éléndue, doil être au moins égal sinon supérieur à celui de cette dernière. Pour ces raisons, il est probable que Vovga n'aurait pas obtenu un résultat aussi brillant dans le Rhône supérieur ên opérant avec des Truites communes.

En résumé, je crois donc pouvoir conclure en disant que, dans les ruisseaux de montagne où, aux causes de destruction banales (ennemis des alevins, cannibalisme, etc.) s'ajoutent souvent de brusques et violentes variations de régime, les repeuplements effectués avec de très jeunes alevins ne dojvent guère laisser espérer un rendement numérique de plus de $10 \%$, mais que, dans certaines conditions particulièrement favorables (eaux plus régulières, nourriture plus abondante), , il est susceptible d'atleindrc jusqu’à $20 \%$.

Ce résultat scmblera sans doute bien médiocre à de nombreuses Sociétés de pêche qui, pour la plupart, ont une idée plus " généreuse " de leurs opérations đe repeuplement. Elles s'en consolenont avec l'espoir qu'il pourra parfois être dépassé et, eñ réfiéchissant que, même à ce taux de ro \%, l'opération est encore avantageuse, puisque avec un millier d'alevins à $540 \mathrm{Fr}$. (prix de 1939) ils pourront obtenir au moins 18 à $20 \mathrm{Kgs}$ de Truite d'une valeur commerciale de 5 a 600 Fr., sans parler du plaisir inestimable que leur a procuré la pêche.

\title{
OBSERVATIONS ET IDFES SUR LE MÉCANISME DE LA FRAYE
}

\author{
Par M. Maunice Vouga, \\ Inspecteur genéral de la Peche, à Neuchatèl (Suisse).
}

\section{INTRODUCTION}

La littérature halieutique s'enrichit chaque année de nombreux ouvrages sur la capture des poissons, la fabrication des mouches, l'art de la pêche au lancer, que sais-je encore ? Les hommes'se donnent vraiment beaucoup de peine pour arriver à détruire les poissons de luxe de nos lacs et de nos rivières ; mais rares, excessivement rares sont les observations apportées : notre bagage scientífique et qui aboutissent à l'amélionation et au développement des divers cheptels de nos eaux.

Il caut être juste pourtant et reconnaître que, pendant des années et des années, les hommes de ma génération ont dú vouer leur principal effort à la mise au point des méthodes de pisciculture adéquates à chaque espèce de poissons. La technique, pour ainsi dire rudimentaire du début du siècle, ₹ fait d'énormes progrès. On sait maintenant comment procéder, exacte- 
ment et dans les plus menus délails, à la pêche et aux soins des géniteurș, à la fécondation artificielle ; on connait les précautions qui doivent être prises pour mener à bien l'incubation des œufs, leur expédition à de longues distances, leur éclosion ; presque tout nous est également connu de cc qui a trait aux soins à donner aux alevins, à leur transport, à la mise ì l'eau, à leur répartition aux endroits favorables. On a appris également ì élever en grand dans des aquariums, dans des bacs, dans des étangs, dans ies ruisseaux sauvages, etc., les sömmerings des principales espèces. Toute cette technique nous est maintenant familière, les aménagistes des eaux douces l'ont bien mise au point et l'ont enseignée à leur personnel de gardes-pêche et de pisciculteurs.

Il paraît à l'auteur de ces lignes qu'une nouvelle orientation s'impose ct qu'il y a lieu, maintenant, de consacrer un peu plus de temps aux pro. blèmes d'économie piscicole proprement dits : établir les coùrbes dé vie des principales sortes de poissons, savoir à quel àge les géniteurs mâles ou femelles se présentent pour la première fớs sur les frayères ct combien dt: fois. au cours de leur existence, ils pourront apporter leur contribution it la multiplication de l'espèce ; quelle est la durée de vie moyenne des hôtes de nos rivières et de nos lacs ; quelles précautions doivent êtrc prises pour éviter que les jeunes et les adolescents ne soicnt capturés avant leurs premières amours; quels sont les aménageménts qui conviennent le mieu x aux divers types de nos eaux ; les équilibres piscicoles les plus rémunéraleurs ; les réactions des poissons sur les poissons ; les tencurs en oxygène indispensables aux diverses espèces; comment se fait la combustion des matières organiques dans nos divers domaines aquicoles; lc développe. ment du phytoplancton et du zooplancton; quelle est leur composition pendant les douze mois de l'année ; quelles sont les fluctuations de leurs courbes, maxima et minima, pendant les décades successives et les conséquences qui en découlent pour la pêche dans les lacs ; quels commentaires enfin suggèrent les statistiques de pêche ? Bref, il y a là, pour les futurs inspecteurs de pêche en tous les pays, des séries de travaux dont l'importance est incalculable ; il convient qu'on s'y attelle couragcusement.

Si l'on veut fermement progresser et créer de toutes pièces une science halieutique, il est indispensable de pousser nos investigations dans ces divers domaines.

\section{Mécanisme de la fraie}

Les naturalistes d'autrefois ont consacré d'admirables pages à la morpho. logie, à la classification des Vertébrés de nos eaux, mais les renseignements qu'ils nous fournissent sur les données plus haut mentionnées et sur le mécanisme de la fraye, entre autres, sont bien rares. Nous savons que les oufs des Truites, ou des Brochets, par exemple, mûrissent dans les grappes ovariennes, puis qu'ils se détachent de ces grappes et demeurent dans lá cavité abdominale jusqu'au moment où, réunis sur les frayères, 
mâles et femelles procéderont à l'érnission des œufs et à leur fécondation. Mais nous ne savons pas si cette fraye est un acte amoureux ou une mise bas, si l'acte de reproduction est entièrement dépendant de leur volonté ou, au contraire, si le déclanchement de l'amour est fonction d'autres facteurs, an parliculier de la température et de la dose d'oxygène dissous.

Quand tout se passe normalement, c'est-à-dire quend les saisons se suivent selon un rythme connu, il nous paraît qué chaque espèce de poissons vient à peu près à dale fixe se livrer sur les frayères à la multiplication de l'espèce et il ne nous semble pas qu'il y ait là matièrè à de longues dissertations. Toutefois, les années se suivent et ne se ressemblent pas, quand nous voyons, dans nos régions tempérées, comme ce fut le cas au printemps 1938, des anomalies considérables dans les températures, nous constatons que tout $n$ 'est pas aussi simple qu'on le pense.

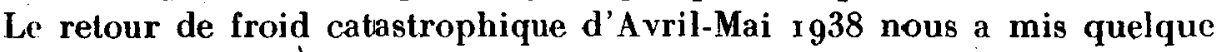
peu sur la voié.

Fn Février-Mars r 938 le temps était superbe, les nuits déjà presque chaudes, la végétation cst partíe comme si le printemps était de retour, les eaux se sont réchauffées également beaucoup plus vite que de coutume. la vigne a " débourré ", comme disent nos vignerons, presque un mois plus lôt que d'habitude, puis le retour de froid est venu et pendant une vingtaine de jours les gelées ont succédé aux gelées anéantissant l'espoir de l'arboriculteur et celui du vigneron.

Et nos poissons, qu'ont-ils éprouvé en l'occurrence ? Ceux qui les ont suivis de près ont vu que, pour eux aussi, ce retour de froid était un vraj désastre. Les Brochets, au début de Mars, dans l'eau déjà tempérée des bords de nos lacs, à $8^{\circ}, 9^{\circ}, 10^{\circ}, 12^{\circ} \mathrm{C}$., selon les places, étaient murs pour lí fraye. Nous avons pu récolter les premiers ocufs et à fin Février les mettre en incubation dans nos piscicultures à eaux chaudes $\left(9^{\circ} \mathrm{C}\right.$. environ) ; mais brusquement le retour de froid fit sentir ses effets. Dans les roseaux, sur les laiches immergées, la température descend très rapidement, retombc à $\xi^{\circ}, 7^{\circ}, 6^{\circ}$ el même plus has dans certaines régions fortement exposées aux courants et au vent du nord. La fraye des Brochets s'arrête net, les captures de géniteurs cessent lotalement. Que deviennent nos poissons en pareille circonstance?

Les mâles abandonnent les places de fraye, ils ne développent plus de laitance ; les testicules regressent rapidement.

Quant aux femelles; leur sort devient tragique, leur ventre est rempli d'ouufs détachés des grappes ovariennes. Donc ces oufs ne sont plus nourris. Peuvent-ils demeurer longtemps dans la cavité abdominale?

L'expérience nous montre que si l'abaissement de la température dure, non plus quelques jours, mais trois ou quatre semaines, la masse des ovules dans la cavité abdominale entre en décomposition et entraîne la mort des génitrices.

Un peu partout, en 1938 , les pêcheurs ont signalé, pourrissant dans les roseaux ou flottant in extremis sur l'eau, de ces femelles de brochets dont ils ne s'expliquaient pas le décès ou l'agonie. En ouvrant leur corps 
il se dégageait une odeur épouvantable provenant de la complète décomposition des ovules, ét il ne s'agissait pas, en l'occurrence, d'ovaires contami nés par des Sporozoaires.

Il nous paraît pouvoir se déduire de cette observation que la fraye des Brochets ne dépend donc pas uniqucrnent de la volonté des poissons mais plutôt que le mécanisme de l'acte amoureux est déclanché probablement. par une hormone qui ñ'est elle-même sécrétćc que si les circonstances sont favorables, c'est-à-dire qu'une bonne température, peut-être aussi un certain pourcentage d'oxygène dissous, sọnt nécessaires à la formation de cette hormone reproductive.

Rapprochons cette observation de r 938 avec d'autres faits constatés antérieurement, soit dans les piscicultures, soit dans les eaux libres

\section{NOMALIES DE LA FRATE}

A. - J'ai déjà expọé ici (I) que, si un pisciculteur négligent ne fraye pas à fond une femelle de Truite et lui laisse, par exemple, une centaine d'œufs, ces oufs, au cours de l'année qui suit, se résorbent petit à petit comme, dans certains cas, chez une femme, le foetus d'une grossesse extrautérine ; il se prọduit une sorte de momification. Tout le contenu de l'ovulte se résorbe et il ne reste en définitive que les coques des œufs qui s'agğlomèrent et forment un bourrelet empêchant, dans bien des cas. l'émission des cufs l'année suivante. Le pisciculteur qui tient en mains une de ces femelles sent que les nouveaux aufs sont bien mûrs, mais, malgré la pression exercée, il ne peut les expulser ; il faut tout le savoir faire d'un vieux praticien pour se rendre compte que l'obstruction est due à cellic cause, et provoquer ensuite une pression assez forte vers l'oviducte pour faire sortir, malgré tout, ce bouchon de coques agglornérées. Ce dégage ment obtenu les nouvelles ovules s'écoulent ensuite normalement. Pour éviter cet ennui j'ai recommandé de passer toujours deux ou trois fois les femelles à la fraye artificielle afin de les débarrasser complètement des derniers œufs qui se trouvent toujours autour des appendices pyloriques. D'après les nombreuses lettres, reçues de tous côtés, je pensé que ce phéno. mène est maintenant bien connu et que les pisciculteurs consciencieux ne commettent plus cette erreur.

Les observations de ce type nous permettent d'énoncer la règle suivante - lorsqu'il demeure, pour une cause ou pour une autre, seulement quelques oufs dans le corps d'une femelle de poisson, ces oxufs se résorbent partiellement sans entrainer la mort de la génitrice.

B. - Dans le lac de Neuchâtel, il y a de grandes Truites lacustres qui alteignent jusqu'à 15 kilogrammes. Ces poissons ne fravent pas au lac mais cherchent, vu leur parenté avec les Saumons, à remonter les cours d'eau

(1) Méthodes et trucs de fécondation artificielle en pisciculture. - Voir Bullétin $n^{\circ} 51$, Septembre 1932, P. $7^{3} ;-n^{0} 52$, Octobre r 932 , p. I 5 . 
pour déposer leur frai dans l'eau vive fortement oxygénée où les alevins à leur najssance pourront saisir au passage la nourriture qui leur est appo: tée par le courant.

Or, il arrive que, selon la sécheresse des hivers, il n'y a pas de crucs favorables jendant les mois d'Octobre, de Novembre et de Décembre. Les Truites qui se tiennent dans le lac généralement au bord du mont, ne sentent pas alors de courant et ne peuvent trouver les rivières, dont le débit est réduit souvent à sa plus simple expression. Comme dans le cas

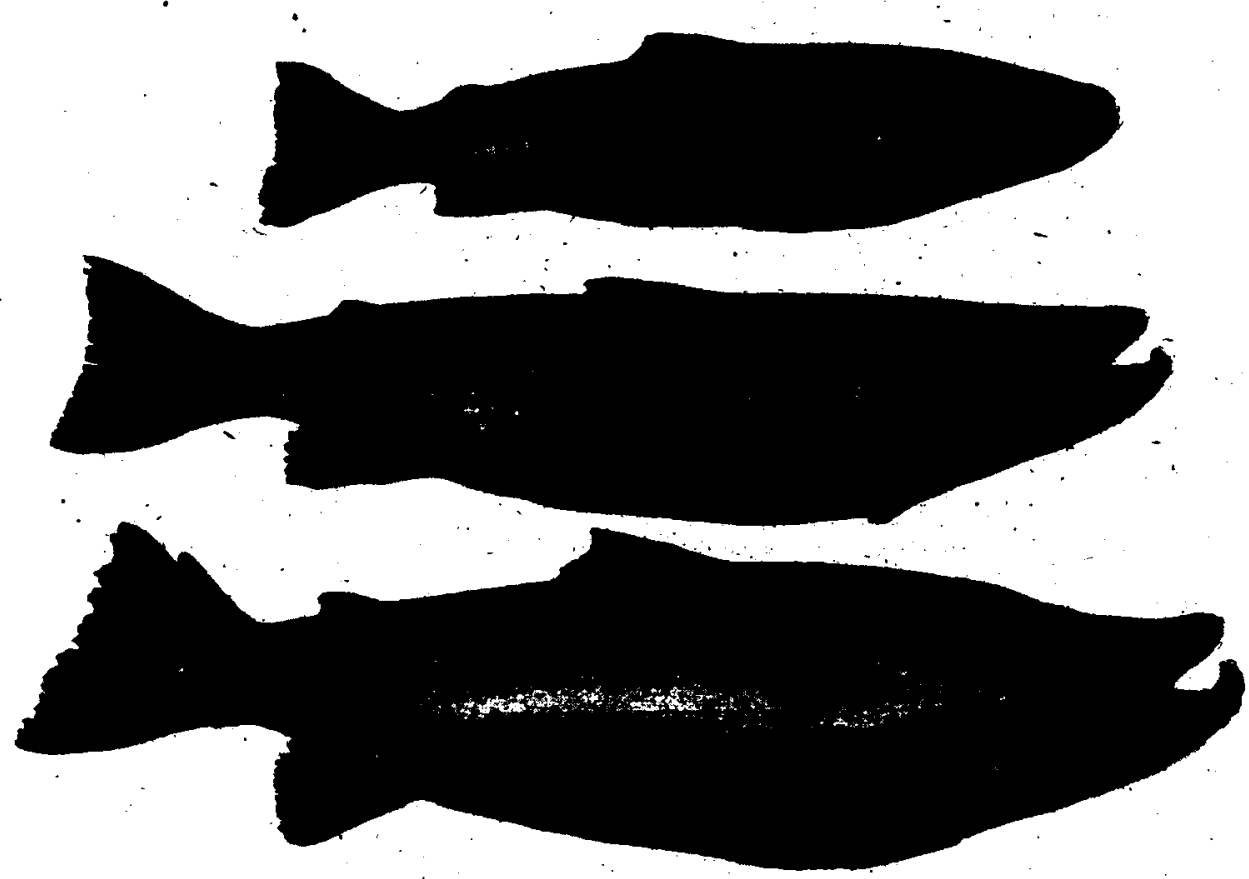

Fic. 1. - Truites de lac mâles en fraye pêchées le 17 Février 1028, soit avec plus d'un mois de retard sur la fraye normale.

En haut, sujet de 5 ans ; longueur, 63 centimetros ; poids, 2.800 grammes.

$\Lambda u$ milieu, sujet de 6 ans ; longueur, 80 centimetres ; poids, 4.700 grammes.

En bas, sujet de 7 ans ; longueur, 84 centimètres; poids, 7 kilogrammes.

des Brochets, - plus haut signalé, - les. Truites femelles sont, vers la fin de Décembre, complètement mûres, leurs ceufs, détachés des grappes ovariennes, demeurent dans la cavité abdominale et ne sont plus nourris. Si la crue de la rivière arrive in exlremis, au début de Janviẹr, màles et femelles sè précipitent dans le courant et en un ou deux jours, laissés à eux-mêmes, ces poissons se débarrassent de la totalité de leurs œuís ; ji par contre nous les capturons pour le service de pisciculture, nous obtenons des femelles qui peuvent être frayées immédiatement sur place, sans stabu lation quelconque dans nos réservoirs ; les aufs sont encore de première qualité. Mais, si la crue ne vient qu'à la fin de Janvier ou à la mi-Février (comme il advint le 17 Février 1928) nous obtenons des femelles à oufs lunés, c'est-à-dire que sur chaque ovule il se forme un disque légèrement 
opaque, signe d'un commencement de résorption du contenu de l'ouf ; ç ovules ne peuvent plus être fécondées et dès qu'elles arrivent en coṇtact avec l'eau, l'albumine qu'elles contiennent se coagule, l'œuf devient complètement blanc et la récolte entière est irrémédiablement perdue (Fig. 1).

Nous constatons donc par ce second exemple que si les Truites de lac ne Irouvent pas dans la nature les conditions favorables à l'ćmission des œufs, il leur est impossible de se débarrasser volontairement de leur frai et il est plus que probable que la décomposition de toute la masse des ovules provoque infailliblement la mort de ces malheureuses génitrices. Dans le présent cas l'hormone d'excitation génésique ne s'est formée, ni chez les mâles, ni chez les femelles, faute probablement d'uue eau suffisamment riche en oxygène pour provoquer sa sécrétion.

C. - Au lac de Montana-sur-Sierre, canton du Valais, j'ai introduit il y a environ une quinzaine d'années, un lot de Truites arc-en-ciel; ces 'Truites, comme toujours, prospérèrent admirablement bien et l'amodiataire de ce lac fit de superbes captures dès la deuxième année. La quatrièmı: année, il me fit venir à Montana pour, m'écrivait-il, " admirer la plus belle frayère de Truites arc-en-ciel qui ait jamais existé en Suisse ". J'accourus aussitôt et effectivement, à l'arrivée du " bisse " (r) dans ce lac, je trouvai réunis 6 à 700 poissons, d'une livre en moyenne, qui semblaient se livrer avec frénésie aux joies de l'amour.

J'assistai, en observateur encharité, à cc spectacle et conseillai à l'amodiataire de prendre quelques-uns de ces poissons pour les frayer artificiellement et mettre les ocufs à l'abri des déprédatcurs, parents y compris, qu . ne manqueraient certainement pas, la fraye terminéc, de manger bonne: partie de ces ocufs. Il en fut fait ainsi ; les résultats obtenus en pisciculture furent excellents, œufs et laitance étant de parfaite qualité: Ceci se passait au mois de Mai. Au mois de Juillet, nouvelle leltre du locataire : "Mes poissons meurent en quantités, prière de venir au plus vite ". Effeclivement, dès le mois de Juillet, nolre homme pouvait ramasser en surface les Truites se traînant lamentablement, à fin de vie, lc ventre complètement ballonné. Je constatai dès mon arrivée que tous les poissons morts ou en train de mourir étaient des femelles qui, n'ayant pas pu frayer, avaien! tous leurs cufs complètement lunés en pleinc décomposition. Tous les mâles se portaient bien.

Que s'était-il passé ? Bien certainement il y avait cu frayc ou tentative de fraye. Certaines fèmelles trouvant à l'arrivée du "bisse " une ean fraîche, bien aérée, un sol de gravier propice et de nombreux mâles, pour les servir, avaient frayé ou essayé de frayer. Nous avions passé des heures à les regarder, mais si certaines avaient réussi à émettre leurs oufs, d'autres n'y étaient pas parvenues. Cette fois-ci l'accident ne dépendait pas du tout

(r) Ruisseau artificiel, provenant des glaciers ct servant à larrosage des prairies et les vignes, en Valais. 
des circonstances locales, mais uniquement du fait que les poissons, provenant. de génitrices frayées artificiellement de génération en génération, avaient complètement perdu l'aptitude à émeltre librement leurs œufs. Le mécanisme musculaire probablement était faussé. Elles auraient bien voulu frayer, les conditions requises étaient là, l'excitation génésique aussi, mais elles ne pouvaient plus faire sortir leurs oufs et, comme dans les cas plus haut cités, la résorption de la totalité des œufs ne pouvant se faire, cette masse d'ovules en décomposition entraînait irrémédiablement la mort.

Nous nous trouvons ici en présence d'un nouvcau cas d'impossibilité d'émission d'oufs, mais dû̀ à une autre cause que précédemment. On peut le rapprocher de celui observẻ chez les poules dont les oufs sont incubés à la couveuse artificielle ; il est connu que sur cent poules obtenues de cette manière-là il n'y en aura que quelques-unes qui éprouveront le désir de couver; les autres ont perdu ce pouvoir et, si on a soin de prendre des œufs de ces poules qui ne couvent plus pour faire de nouvelles générations de poulettes éclosant artificiellement, on obtiendra bientôt une race de poules ne sachant plus du tout ce qu'est la maternité due à la couvaison. Nous avons fait la même abservation sur des faisans de chasse élevés en volière. Nous obtenons. bien des oeufs, même beaucoup d'œufs, mais les faisanes mises on liberté ne construisaient plus de nid et ne couvaient plus.

D. - Lorsque nous avons entrepris, en 1939, la grande expérience d'acclimatation des Truites ác-enciel dans les eaux vierges du Rhône supérieur ( $\mathrm{r}$ ), nous nous sommes servis de 120.000 alevins provenant de piscicultures de cinq ou six régions différentes de la Suisse, Ces alevins produisirent un cheptel d'envinon 40.000 Truites sur lesquelles il nous fut possible, pendant dix ans, de faire d'intéressantes observations. Il y a lieu de relever ici qu'au bout de la troisième année de l'immersion nous trou. vâmes déjà un certain nombre de femelles capábles d'émettre des ceufs . le lot des génitrices augmenta d'année en année, certaines femelles n'étant mûres qu'au bout de la quatrième, de la cinquième ou même de la sixième année. N'oublions pas que nous avions à faire ici à un mélange de toutes les races d'arc-en-ciel introduites en Europe.

Nos pêches d'essai nous firent constater qu'un certain nombre $d e^{\text {. }}$ femelles, trouvant dans les eaux du Rhône supérieur toutes. les conditions requises pour-l'excitation génésique, émettaient normalement leurs cufs. Toutefois, au cours des pêches d'automne, il nous arriva fréquemment, en autopsiant nos captures; de trouver dans la cavité abdominale un certain nombre d'œufs qui n'avaient pu être évacués et étaient en voie de résorption. D'autres femelles n'ayant pu, vu leur mécanisme de fraye faussé par la pisciculture artificielle de leurs parents, émettre leurs ceufs, furent tron-

(r) Voir Bulletin. $=\mathrm{n}^{\circ} 43$, Janvier 1932, p. $217 ;-\mathrm{n}^{\circ} 50$, Aơt 1932 , p. 58 ; $n^{\circ} 64$, Octobre rg33, p. 102; - $n^{\circ} 67$, Janvier 1934, p. r95; - no 83, Avril r $9^{35}$, p. $34 \mathrm{r} ;-\mathrm{n}^{\circ} 83$, Mai $\mathrm{r} 35$, p. 265 . 
vées péries, mais ce fut un très petit nombre. Ictuellement, il s'est formé, au Rhône supérieur, un nouveau lot, issu de ces premières génitrices, qui est maintenant capable de frayer nalurellement, normalement, comme tous les poissons sauvagr's et nous ne trourons plus, lors des autopsies que. par-ci par-là, un ou deux aufs retenus dans les appendices pyloriques.

De cette observation, nous pouvons conclure que, si le mécanisme de la fraye peut se perdre lorsque les génitcurs sont de génération en génération fécondés artificiellement, il peut aussi se récupérer par le relour à l'état normal après avoir, bien entendu, enregistré des cas mortels dus à l'impos. sibilité totale d'accoucher.

E. - Je rappelle ici simplement, pour mémoire, tant le cas est bicn connu de tous les carpiculteurs, que la fraye des Carpes ne se fait qu'à des conditions de température déterminées et chacun sait également que, dans les caux qui n'atteignent pas cette température critique, la reproduction de la Carpe est impossible. Nous pouvons donc en déduire que l'hormonr. qui déclanche l'excitation génésique de la Carpe n'est produite que lorsqu: la température atteint lo degré favorable.

F. - Au lac de Neuchâtel, le petit Corégone de fond, la "Bondelle " Coregonus exiguus a, pendant le début de mes années d'inspectorat, toujours frayé aux environs du I 2 Janvier. La fraye était considérée comm. terminée le zo et l'ouverture de la pêche étail fixée par la loi au 26 . Janvier. Quelle ne fut pas notre surprise lorsque le $?_{7}$ Janvier rg28, les pêcheurs, en relevant leurs filets, les trouvèrent blanes de poissons : ils ne purent les relever tous le même jour et, du 27 au 3 r Janvier, il fut capturé 34.or 2 kilo: de Bondelles, soit le tiers environ de la totalité de la capture d'une annéc normale. D'où provenait ce retard inusité dans la fraye $P$ Nous fûmes pendant des années à nous le demander ; ce n'est que cclle année-ci, en 1939 , donc après onze ans d'essais divers et d'expériences nouvelles, que nous eûmes la clef de ce mystère. On croyait jusqu'alors, d'après des données généralement admises, qu'un lac comme le lac de Neuchâtel, du type oligotrophe, avait toujours dans ses couches profondes une tencur en oxygèn. voisine de la normale. Or, nos observations de ces dernières années nous: firent douter de cet ćtat de choses et nous entreprîmes, avec l'aide de M. E. SEvaud, assistant au Laboratoire de l'Institut de botanique de I'Lniversité de Neuchâtel, une série de dosages d'oxygène dans toutes les couchen du lac de Neuchâtel, et à de multiples endroits. Ces déterminations faites d'après les méthodes de Horer et de Winkuer, nous firent constater que, certaines années, ensuite de brassages insuffisants, manque de vagues ou températures ańormalement chaudes, le dosage d'oxygène tombait pendant des semaines aux environs de 2 centimètres cubes par litre. Or, il va de soi que des poissons de la famille des Salmonides, à opercules largement ouverts et ayant toujours besoin de fortes quantités d'oxygène, ne peuvent frayer, à même le fond, si la teneur en oxygène est notoirement insuffisante. d'où retardement de la fraye jusqu'au moment où le vent du nord-est 
"la bise " agitant fortement le lac et le refroidissant, provoquent la formation de forts courants lacustres très oxygénés et le retour à l'état normal. Alors, mais sculement alors, les Bondelles trouvent dans le fond du lac les conditions favorables à la multiplication de l'espèce, la sécrétion de l'hormone sexuelle provoque le désir génésique et la fraye peut se faire.

Nous pouvons rapprocher ces observations de celles déjă publiées par le célèbre professeur Roule sur la reproduction des Saumons qui fait infailliblement choisír aux géniteurs de cette espèce remontant les fleuves de France, les affluents aux eaux particulièrement riches en oxygène, laissant soigneusement de côté les affluents de droite ou de gauche dont la teneur en oxygène n'est pas suffisante.

\section{Conclusions}

Il nous paraît possible de tirer de ces observations (qui n'ont pas encore la prétention d'être absolument parfaites, mais qui doivent à notre point de vue orienter et guider de nouveaux' chercheurs) les conclusions suivantes :

L'acte d'amour de certains poissons de nos eaux douces d'Europe n'eśt pas uniquement dépendant de la volonté du poisson. Ce n'est pas une recherche pure et simple des sexes qui a lieu lorsque les éléments de repro. duction, laitance et ovules, sont arrivés à maturité. Le déclanchement de l'acte amoureux. ne peut se faire que si les conditions requises sont là. Dans la pratique, neuf fois sur dix, ces conditions : température, oxygéna.. tion de l'eau, se trouveront synchroniquement réunies lorsque les poissons arriveront à maturité sexuelle, puisque ce.sont ces facteurs de température et d'oxygénation qui provoquent la maturité des organes génitaux. Mais Iorsque les concordances habituelles viennent inopinément à manquer, ley femelles de certaines espèces de poissons deviennent incapables de se débarrasser de leurs cufs, faute dé l'excitation hormonique nécessaire. Les males pouvent résorber sans inconvénient leur laitance, les femelles sont dans l'impossibilité de résorber la totalité des ovules et cette rétention entraîne fréquemment la mort des génitrices.

\section{Expérience sur les épinoches.}

Si l'un de mes collègues ou un Directeur dé laboratoire de zoologie lacustre disposait des moyens et du temps nécessaires, je lưi proposerais de tenter l'expérience suivante, sans doute féconde en enseignements . Environ quinze jours avant la fraye, mettre dans une dizaine d'aquariums, sablés, herbés d'Elodéas, de Renoncules, de Fontinales, etc., et de débris de végétaux, un Epinoche mâle et deux femelles gravides. Nourrir comme de coutume avec des Daphnies et quelques Tubifex. Aérer à la pompe par diffuseur habituel. Donc toutes choses égales d'ailleurs.

Puis disposer ces dix aquariums de manière à ce que le numéro I soit toujours à la témpérature de 2 degrés centigrades ; le numéro 2 à 4 degrés ; 
le numéro 3 à 6 degrés ; le numéro 4 à 8 degrés; le numéro 5 à ro degré: et ainsi de suite jusqu'au numéro ro dont l'eau sera maintenue pendant toute la durée de l'expérience à 20 degrés.

Observer ce qui se passera ; préparer un lot de poissons de remplaçement car. dans certains cas, la mortalité sera inévilable ; constater quand et comment se fera la construction des nids, quand aura lieu la ponte et dans quels aquariums les naissances viendront à bien.

$\Lambda$ près ces essais, je crois que nous aurons des idées nouvelles sur la multiplication des poissons cn eaux libres et je pense aussi qu'on s'expliquera la disparition de géniteurs restée mystérieuse jusqu'à ce jour.

Je fournirai volontiers les Epinoches qui, cela va de soi, devront provenir d'caux publiques (canaux du Valais, par exemple).

\title{
LA DISTRIBUTION GÉOGRAPHIQUE IDU · SAUMON DU PACIFIQUE
}

(Genre Onchorhynchus)

\section{INFLUENGE SUR ELLE DES CONDITIONS DU MILIEU}

\author{
Par Frederick A. DAVIDSON, \\ Docleur en Phi orophie \\ et Samuer A. HUTCHINSON, \\ Bio ogistes de l'United Stales Bureau of Fisheries (1).
}

\section{INTRODUCTION}

Il existe cinq espèces principales du gen re Onchorhynchus Suckley, qui est le Saumon du Pacifique, ce sont : - le chinook ou quinnat : Saumon de Californie, (O. tschauytscha Walbaum); - le sockeye ou red : Sau-

(1) Bien que ce travail concerne un genre de poisson dont l'acclimatation en liurope n'a pas été couronnée de succès, it présente un intérêt général incontestable par les données qu'il fournit sur les exigences biologiques de Salmonidés migrateurs dont les besoins essenticls ne doivent pas différer sensiblement de ceux de leur parent de l'Atlantique : Salmo salar $L$.

M. Charles E. Jacksos, Icting Commissioner de lt. S. Lureau of Fisheries a bien voulu, par lettre $n^{\circ} 800 \mathrm{du} 28$ juin 1939 , autoriser la publication par le Bulletin d'ume traduction de l'étude de MM. I) svinsox el Hutchissox. Qu'il ell soit ici sincèrement remercié.

Cette traduction est libre et quelque peu abrégée. Les photogravures qui illustronl le texle américain n'ont pas ćté reproduites, mais sculement les cartes.

L'original forme le fascicule $n^{\circ} 26$ du tome XLVIII du Nu!letin of the Bureau of Fisherics, pp. 667-693 ; - Government Printing Office, Washington D. C., sg38. Lo titre est : - The geographic distribution and environmental limitations of the Pacific Salmon (genus Onchorhrnchus). 